\title{
Identification and characterization of microRNAs in the ovaries of multiple and uniparous goats (Capra hircus) during follicular phase
}

\author{
Ying-Hui Ling ${ }^{1,2+}$, Chun-Huan Ren ${ }^{1,2+}$, Xiao-Fei Guo ${ }^{1,2}$, Li-Na Xu ${ }^{3}$, Ya-Feng Huang ${ }^{1,2}$, Jian-Chuan Luo ${ }^{1,2}$, \\ Yun-Hai Zhang ${ }^{1,2}$, Xiao-Rong Zhang ${ }^{1,2^{*}}$ and Zi-Jun Zhang ${ }^{1,2^{*}}$
}

\begin{abstract}
Background: Superior kidding rate is an important economic trait in production of meat goat, and ovulation rate is the precondition of kidding rate. MicroRNAs (miRNAs) play critical roles in almost all ovarian biological processes, including folliculogenesis, follicle development, follicle atresia, luteal development and regression. To find out the different ovarian activity and follicle recruitment with miRNA-mediated posttranscriptional regulation, the small RNAs expressed pattern in the ovarian tissues of multiple and uniparous Anhui White goats during follicular phase was analyzed using Solexa sequencing data.

Results: 1008 miRNAs co-expressed, 309 and 433 miRNAs specifically expressed in the ovaries of multiple and uniparous goats during follicular phase were identified. The 10 most highly expressed miRNAs in the multiple library were also the highest expressed in the uniparous library, and there were no significantly different between each other. The highest specific expressed miRNA in the multiple library was miR-29c, and the one in the uniparous library was miR-6406. 35 novel miRNAs were predicted in total. GO annotation and KEGG Pathway analyses were implemented on target genes of all miRNA in two libraries. RT-PCR was applied to detect the expression level of 5 randomly selected miRNAs in multiple and uniparous hircine ovaries, and the results were consistent with the Solexa sequencing data.

Conclusions: In the present study, the different expression of miRNAs in the ovaries of multiple and uniparous goats during follicular phase were characterized and investigated using deep sequencing technology. The result will help to further understand the role of miRNAs in kidding rate regulation and also may help to identify miRNAs which could be potentially used to increase hircine ovulation rate and kidding rate in the future.
\end{abstract}

Keywords: MicroRNA, Kidding rate, Solexa sequencing, Ovary, Follicular phase, Goat

\section{Background}

MicroRNAs (miRNAs) are a group of endogenous $\sim 22 \mathrm{nt}$ small non-coding RNAs that can modulate gene expression by inhibiting mRNA translation or regulating mRNA degradation at the post-transcriptional level [1]. It was once estimated that known miRNAs account for around $1 \%$ of predicted genes in higher eukaryotic genomes and

\footnotetext{
* Correspondence: zhangxiaorong01@163.com; zhangzijun6666@163.com ${ }^{\dagger}$ Equal contributors

${ }^{1}$ College of Animal Science and Technology, Anhui Agricultural University, No. 130 Changjiang west road, Hefei 230036, P.R. China

${ }^{2}$ Anhui Provincial Laboratory of Local Animal Genetic Resources Conservation and Biobreeding, No. 130 Changjiang west road, Hefei 230036, P.R. China Full list of author information is available at the end of the article
}

that up to $30 \%$ of genes might be regulated by miRNAs [2]. MiRNAs regulation have been implicated in varied physiological processes including cell proliferation [3], differentiation [4], apoptosis [5], tumorigenesis [6], hormone secretion [7], metabolism [8] and reproduction control [9]. On goat's miRNA study, researchers have focused their interest on mammary gland of dairy goat [10], hair follicle of cashmere goat [11] and muscle or reproduction of meat goat $[12,13]$. A recent study showed that over-expression of miR-103 in goat's mammary gland epithelial cells increased transcription of genes associated with milk fat synthesis, resulted in an up-regulation of fat droplet formation, triglyceride accumulation, and the proportion of unsaturated fatty acids [14]. MiRNAs research 
in animal ovary has been extensively explored with the improvement of study methods. MiRs-31 and MiRs-92 were discovered in pig ovary by homology analysis and confirmed with northern blot [15]. Cloning technique had been adopted to identify miRNAs expressed in mice ovary, obtained a total of 122 miRNAs from the ovaries of 2-wk-old and adult mice [16]. A mouse mutant with a $\sim 75 \%$ loss of Dicer1 miRNA levels was predicted to cause the decreasing of angiogenesis in the corpus luteum ultimately resulted in female infertility. On further study, the miR-17-5p and let-7b which regulate the expression of tissue inhibitor of metalloproteinase 1 had been proved contributed to the mutant mouse's infertility [17]. In 2013, the different expression of miRNAs in the ovaries of pregnant and non-pregnant Anhui White goats was identified and analyzed, 617 conserved and 7 putative novel miRNA in hircine ovaries had been detected by high-throughput sequencing [13].

Superior kidding rate is an important economic trait in production of meat goat, while ovulation rate is the precondition of kidding rate [18]. Follicular phase accompanied by the increasing of FSH, is the phase including of follicle recruitment and dominant follicle development. Multiple follicle development and ovulation have been attributed to higher elevations of FSH above the threshold level [19-21]. Researchers had discovered that bone morphogenetic protein 15 (BMP15) and growth differentiation factor 9 (GDF9) contribute to all stages of follicular development including activation of the primordial follicles $[22,23]$. James et al. mutated the genes for oocyte-derived growth factors GDF9 and BMP15 which were associated with both increased ovulation rate and sterility in Cambridge and Belclare sheep [24,25]. There are also specific genes, for example the FecB or Booroola gene, that result in ovulation rates greater than five $[18,26]$. In recent years, many studies indicated that miRNAs play critical roles in almost all ovarian biological processes, including folliculogenesis, follicle development, follicle atresia, luteal development and regression [17,27-31]. The Anhui white goat is known for its precocious puberty, higher fertility, and higher leather quality compared with other types of goat. Anhui white goat ewes can estrus all year round. The average Anhui white goat kidding rate is $227-239 \%$ which is belong to varieties of high kidding rate in goats. It is therefore an ideal model for the study of goat breeding traits.

In the present study, we characterized and investigated the differential expression of miRNAs in the ovaries of multiple and uniparous Anhui White goats using deep sequencing technology. The result will help to further understand the role of miRNAs in kidding rate regulation and also may help to identify miRNAs which could be potentially used to increase hircine ovulation rate and kidding rate in the future.

\section{Results}

\section{Overview of sequencing data}

In order to identify differentially expressed miRNA during follicular phase in the ovaries of multiple and uniparous Anhui White goats, two small RNA libraries were constructed by Solexa sequencing. A total of 12,000,000 raw reads were obtained. After discarding the sequences shorter than $18 \mathrm{nt}$, eliminating low-quality sequences and removing contaminants formed by adapter-adapter ligation, reads without 3'ligation and insert tags were obtained. Ultimately, 5,948,837 and 5,945,145 clean reads which obtained from multiple and uniparous goats remained for further analysis (Table 1). Subsequently, all identical sequence reads were classified as groups, and 160,284 and 235,735 unique sequences were obtained (Additional file 1). The length distribution of the reads was similar between the two libraries (Figure 1). The majority of the small RNA were 20-24 nt range. Sequences $22 \mathrm{nt}$ in length, the typical size of Dicer-derived products [32], peaked at length distribution, respectively accounted for $56.93 \%$ and $54.26 \%$ of the total sequence reads in the multiple and uniparous libraries.

For assessing the efficiency of Solexa sequencing and the quality of sequence itself, all of the clean reads were annotated and classified by aligning against the Rfam10.1 database, Genbank and the miRBase20.0 database. However, some sRNA tags may be mapped to more than one category. To make every unique small RNAs mapped to only one annotation, we followed the following priority rule: rRNAetc (Genbank $>$ Rfam) $>$ known miRNA $>$ repeat $>$ exon $>$ intron [33]. All of the clean reads were divided into the following categories: exon_antisense, exon_sense, intron_antisense, intron_sense, miRNA, rRNA, repeat, scRNA, snRNA, snoRNA, srpRNA, tRNA, unan (sequences were not mapped to any known reference databases). The composition of the RNA classes in each library was shown in Figure 2 and Additional file 1. The proportion of total rRNA is a mark for sample quality check. Usually it should be less than $60 \%$ in plant samples [34] and $40 \%$ in animal samples as high quality (unpublished data by BGI). The proportion of total rRNA was $2.86 \%$ and $5.05 \%$ in multiple and uniparous librariy respectively, indicating that the ovaries samples collected were of high quality in this study. In order to analyze the two libraries expression and distribution, all of the clean Solexa reads were mapped to the goat genome sequence using SOAP software. In the clean reads of multiple and uniparous libraries, 4,780,962 reads (account for 80.37\%) and $4,705,068$ reads (account for 79.14\%) were mapped to the goat genome (Additional file 1). Conserved miRNAs accounted for $92.28 \%$ and $87.26 \%$ of the total clean reads, and accounted for $20.74 \%$ and $15.63 \%$ of the unique reads (Figure 2) in the multiple and uniparous small RNA libraries, respectively. The result of two libraries showed that 
Table 1 The classification of total small RNA tags by Solexa sequencing

\begin{tabular}{lccccc}
\hline Type & \multicolumn{2}{c}{ Mul } & & Uni & Total \\
\cline { 2 - 4 } & Counts & Percent & & Counts & Percent \\
\hline Total_reads & $6,000,000$ & & $6,000,000$ & $100 \%$ & $12,000,000$ \\
High_quality & $5,965,487$ & $100 \%$ & $5,963,795$ & $1,929,282$ \\
3'adapter_null & 973 & $0.02 \%$ & 1,066 & $0.02 \%$ & 2,039 \\
Insert_null & 354 & $0.01 \%$ & 665 & $0.01 \%$ & 1,019 \\
5'adapter_contaminants & 9,183 & $0.15 \%$ & 10,495 & $0.18 \%$ & 19,678 \\
Smaller_than_18nt & 6,119 & $0.10 \%$ & 6,375 & $0.11 \%$ & 12,494 \\
PolyA & 21 & $0.00 \%$ & 49 & $0.00 \%$ & 70 \\
Clean_reads & $5,948,837$ & $99.72 \%$ & $5,945,145$ & $99.69 \%$ & $11,893,982$ \\
\hline
\end{tabular}

the majority of total reads was classified as miRNA, which suggested that the sequencing of present study was successful. However, the highest fraction of unique reads was attributed to unann, and the length distribution of its small RNA was mainly round about 20-24 nt (Additional file 2). Therefore, just like which had be reported before that there are still many kind of miRNA waiting to be found for us [13].

\section{Differential expression of conserved miRNAs in the ovaries} of multiple and uniparous goats during follicular phase

Since there is no miRNAs information of goat in the miRbase 20.0 database, we aligned the clean reads to the miRNA precursor/mature miRNAs of all known animals in the miRBase 20.0 database. The sequence and count of families (no specific species) were obtained which could be found in the two libraries (Additional file 3). Considering little mismatches between sequences, 1317 and 1441 conserved miRNAs were identified in the multiple and uniparous libraries. Among them, 1008 miRNAs were co-expressed, 309 and 433 miRNAs were specifically expressed in the multiple and uniparous libraries, respectively. The overwhelming majority of these specifically expressed miRNAs' expression level were very low (under 5), whereas the expression level of 4 (miR-29c, miR-1996b, miR-3135b, miR-3934-5p) and 9 (miR-6406,

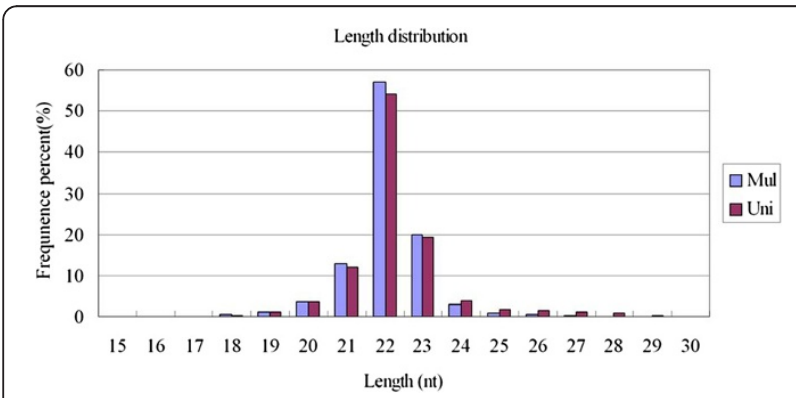

Figure 1 Frequency distribution of sequence lengths of the sequencing results.
miR-6317, miR-4001e-3p, miR-1692, miR-6215, miR-4674, miR-1591-3p, miR-4090-3p, miR-1589) specific expressed miRNAs in multiple and uniparous libraries were higher than 1000 (Table 2). The highest specific expressed miRNA in multiple library was miR-29c which reached counts of 5,214 (normalized expression level of 876), and the highest specific expressed miRNA in uniparous library was miR6406 which reached counts of 42,571 (normalized expression level of 7,161).

The differentially expressed miRNAs between the two libraries were showed in Figure 3 and Additional file 4 . The same as previous study on miRNA detecting $[10,11,13,27,35,36]$, most of its expression quantity were equivalent, while there were also some miRNAs expressed differently between the two experimental group (Figure 3). $60.7 \%$ of the miRNAs expression was not significant, $3.6 \%$ of the miRNAs were significantly different $(0.01 \leq \mathrm{p}<0.05)$ and $35.7 \%$ of the miRNAs were significantly different $(\mathrm{p}<0.01)$ in the multiple and uniparous libraries. The 10 most highly expressed miRNAs (let-7b, let-7b-5p, let-7-5p, let-7c, let-7c-5p, let-7f-5p, let-7f, let-7, miR-140, miR-320a) in the multiple library were also the highest expressed in the uniparous library, and there were no significantly different between each other.

MiRNAs clustered together for similar expression patterns [37]. In Additional file 5, green indicates that the miRNA has higher expression level in multiple library, red indicates that the miRNA has higher expression in uniparous library. All differentially expressed miRNAs in two libraries clustered together after 6 rounds of clustering.

\section{Identification of potential novel miRNAs}

The characteristic hairpin structure of miRNA precursor can be used to predict novel miRNA. The novel miRNAs were predicted by Mireap software (http://sourceforge. net/projects/mireap) through mapping the precursor to goat genome sequences. Novel miRNAs presumed by exploring the secondary structure, the Dicer cleavage site and the minimum free energy of the unannotated small RNA reads. 35 potential novel miRNAs were detected in 
A
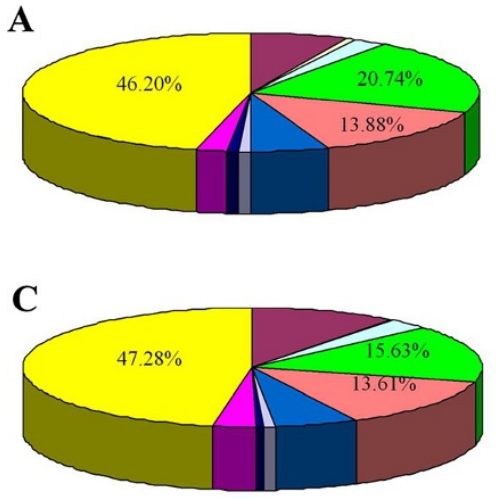

$\square$ exon_antisense $\square$ exon_sense

$\square$ miRNA

- snoRNA

$\square$ rRNA

$\square$ IRNA

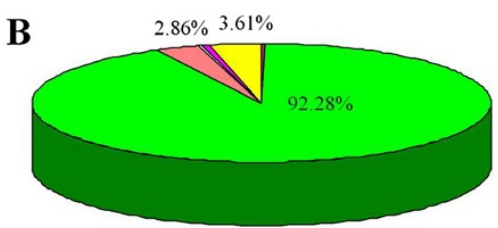

D

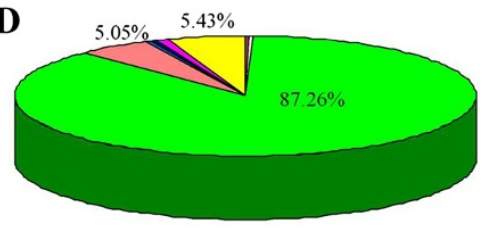

$\square$ intron_antisense $\square$ intron_sense

$\square$ repeat $\square$ snRNA

Figure 2 Composition of small RNA classes of the Solexa. Note: (A) Total number of unique sequences in the multiple library. (B) Total number of reads in the multiple library. (C) Total number of unique sequences in the uniparous library. (D) Total number of reads in the uniparous library.

total, of which 8 potential novel miRNAs were coexpressed, 9 and 18 potential novel miRNAs were specifically expressed in the multiple and uniparous libraries, respectively (Additional file 6 and Additional file 7). The length of the novel miRNA sequences also ranged from 20 to $24 \mathrm{nt}$, and these novel miRNAs were not analyzed further, as their expression quantity were very low.

\section{Target gene prediction for miRNAs}

miRNAs modulate gene expression by inhibiting mRNA translation or regulating mRNA degradation at the posttranscriptional level based on pairing between the $5^{\prime}$ end of the miRNA (i.e., 2-8 nt, the"seed"region) and the 3' untranslated regions (3' UTR) of target mRNAs [1,38-40]. Mireap software was used to predict target genes of the miRNA by searching the goat reference gene database (http://www.ncbi.nlm.nih.gov/genome/10731). In

Table 2 The expression level of specific expressed miRNAs which were higher than 1000

\begin{tabular}{|c|c|c|c|c|c|}
\hline \multicolumn{3}{|c|}{ Mul } & \multicolumn{3}{|c|}{ Uni } \\
\hline MiRNA & Counts & Mul-std. & MiRNA & Counts & Uni-std. \\
\hline miR-29c & 5214 & 876 & miR-6406 & 42571 & 7161 \\
\hline miR-1996b & 1771 & 298 & miR-6317 & 5655 & 951 \\
\hline miR-3135b & 1508 & 253 & miR-4001e-3p & 3644 & 613 \\
\hline \multirow[t]{6}{*}{ miR-3934-5p } & 1212 & 204 & miR-1692 & 3028 & 509 \\
\hline & & & miR-6215 & 2394 & 403 \\
\hline & & & miR-4674 & 2030 & 341 \\
\hline & & & miR-1591-3p & 1991 & 335 \\
\hline & & & miR-4090-3p & 1800 & 303 \\
\hline & & & miR-1589 & 1395 & 235 \\
\hline
\end{tabular}

Note: -std. represents normalized expression level of miRNA in a library. the multiple library, 3,091,082 target sites in 22,171 target genes were predicted for 1,317 conserved miRNAs, and 41,781 target sites in 17,714 target genes were predicted for 17 novel miRNAs. In the uniparous library, $3,427,044$ target sites in 22,171 target genes were predicted for 1,441 conserved, and 67,990 target sites into 19,812 target genes were predicted for 26 novel miRNAs.

\section{Gene Ontology (GO) enrichment and KEGG pathway analysis of target genes}

GO is an international standarized classification system for gene function, which supplies a set of controlled vocabulary to comprehensively describe the property of genes and gene products. There are 3 ontologies in GO: cellular component, molecular function and biological process. The basic unit of GO is GO-term, each of which belongs to one type of ontology. In this study, GO enrichment analysis was used for predicting candidate target genes of all detected miRNAs. In Additional file 8, GO enrichment for gene background based on the cellular component showed that 13,973 genes were mapped to GO terms in the database (http://www.geneontology.org/). For all miRNAs target genes of multiple and uniparous goats in the ovaries during follicular phase, there were 11,577 and 12,767 target genes mapped to the GO terms of cellular component. Compared to the reference gene background, 2 and 4 GO terms were significantly (P-value $<0.05)$ enriched for multiple and uniparous libraries respectively based on the cellular component. Analysis of molecular function showed that 13,013 genes were assigned different functions based on gene background, while 10,806 and 11,902 target genes were involved for multiple and uniparous libraries. Compared to the reference gene background, 11 and 16 GO terms were 


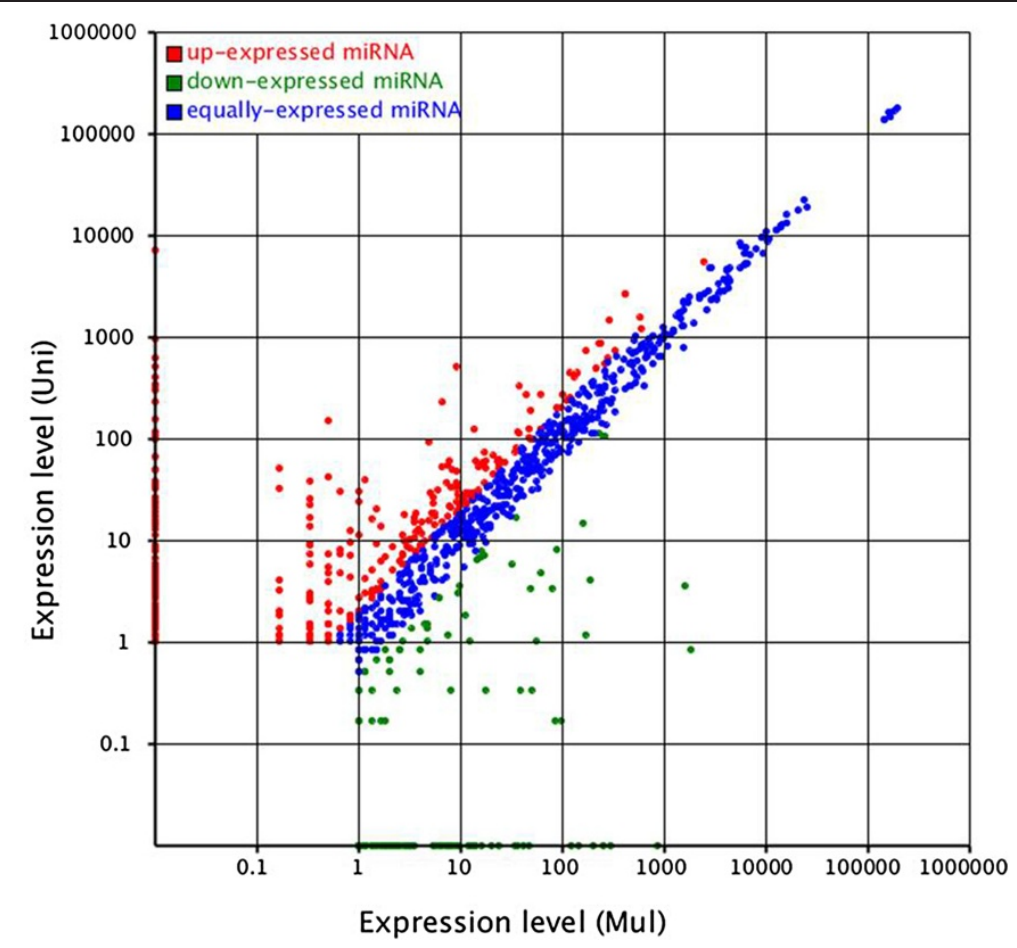

Figure 3 Differences of miRNA expression between the two libraries. Note: The scatter plot of differentially expressed miRNAs (control: $X$-axis, treatment: $Y$-axis). The $X$ and $Y$ show the expression level of miRNAs in the two samples respectively. Red points represent miRNAs with ratio $>2$; Blue points represent miRNAs with $1 / 2<$ ratio $\leq 2$; Green points represent miRNAs with ratio $\leq 1 / 2$. Ratio = normalized expression of the treatment/normalized expression of the control.

significantly (P-value $<0.05)$ enriched for multiple and uniparous libraries respectively based on molecular function. For the reference gene background, 13,113 genes were related to biological processes. However, 10,877 and 11,969 target genes were related to the biological processes of GO terms for multiple and uniparous libraries. Compared to the reference gene background, 1 and 4 GO terms were significantly $(P$-value $<0.05)$ enriched for multiple and uniparous libraries respectively based on biological processes.

KEGG pathway annotation showed that 16,155 background genes were annotated for 309 biological functions. However, 13,472 and 14,860 target genes were annotated to the relevant biological functions for multiple and uniparous goats in the ovaries during follicular phase. The only over-represented miRNA targets belonged to the olfactory transduction pathways in uniparous library, while there 10 pathways were significantly $(\mathrm{P}$-value $<0.05$ ) enriched in multiple library (Additional file 9).

\section{Quantitative RT-PCR validation}

The expression levels of 5 (miR-378a, miR-10a, miR-202$5 p$, miR-84a, and let-7d-5p, and 3 of them were differentially expressed) randomly selected miRNAs were verified in the ovaries of multiple and uniparous goats during follicular phase using RT-PCR. The relative expression levels of 5 selected miRNAs were consistent with the Solexa sequencing results since they had a similar trend of expression in two libraries (Figure 4).

\section{Discussion}

Economic efficiency of a flock of goats is dependent on its total productivity, and the productivity is more dependent on fertility and prolificacy of the female goats than any other components [41-43]. However, hircine fecundity is relatively low [13], and the trait is difficult to improvement by conventional breeding methods for its low heritability which ranged 0.09 to 0.14 based on the study of Zhang et al. [43]. Therefore, researchers pin their hope on molecular assisted breeding technology, and the research of miRNA in reproduction thrived $[2,9,13,27,35]$. In this study, we sequenced the small RNAs in the ovarian tissues of multiple and uniparous Anhui White goats during follicular phase by Illumina Solexa technology, then analyzed the differentially expressed miRNAs, predicted novel miRNAs, and made GO enrichment and KEGG pathway analysis of target genes in two miRNA libraries.

Compare to researches carried by predecessors [36], more conserved miRNAs (1317 and 1441 in the multiple and uniparous libraries) were identified in this study. There are two principal reasons contributing to these 


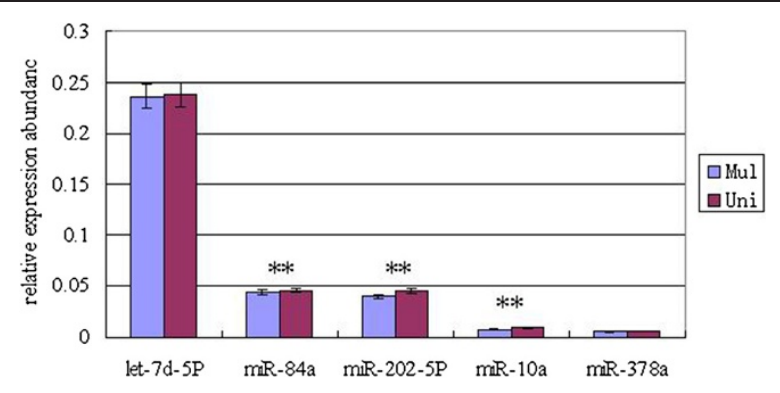

Figure 4 RT-PCR validation of miRNAs identified in goat ovaries using Solexa sequencing technology. Note: **indicate the significant $(P<0.01)$ difference in expression level between multiple and uniparous goats by GLM of SAS software.

results. The first reason is that the detected clean reads was aligned to the latest database of miRBase 20.0 released in June 2013, while 3355 new hairpin sequences and 5393 new mature microRNAs from around 40 new publications were added to the database based on miRBase 19.0, increasing the totals to 24521 hairpin sequences and 30424 mature sequences in all (http://www.mirbase. $\mathrm{org} / \mathrm{blog} /$ ). And the $\mathrm{miR} / \mathrm{miR}^{*}$ nomenclature is finally replaced by the $-5 p /-3 p$ nomenclature after miRBase 18.0, the result leading to miRNA* which was not reckoned in real conversed miRNA once, now named $-5 p$ or $-3 p$; ultimately duplex-like miRNA:miRNA* what was regarded to be one miRNA turned out to be two. The second reason is that the two libraries were compared with all animals in the miRBase 20.0 for there is no miRNAs information of goat in it, and the reference data was species widely.

In ovaries between multiple and uniparous goats of follicular phase, 35 novel miRNAs were predicted in total, which is distinctly more than the amount predicted in our previous study (ovaries from pregnant and nonpregnant goats) implemented by our team workers, Zhang et al. [13]. For this result, a central factor is should be attributed to the new reference of goat genome which published in December 2012 when the study of Zhang et al. had been completed, and they simply identified novel miRNAs by means of alignment with goat expressed sequence tags (ESTs). It worth to point out is that 1 (from multiple libraries) of the 35 novel miRNAs sequence is consistent with the sequence (from non-pregnant libraries) predicted in our previous study. This result may hint that the sequence should be a greater likelihood of a potential new miRNA.

In sheep, selection for superior lambing rate has been showed to alter ovulation rate primarily [44]. And it has been suggested that increased ovulation rates could be due to a wider window of time for follicle recruitment or an increase in the numbers of follicles recruited in ovaries [45]. To find out the different ovarian activity and follicle recruitment in multiple and uniparous goats of follicular phase, differentially expressed miRNA were identified in the two constructed libraries. The 20 most highly expressed miRNAs in the multiple library were mainly consistent with that in the uniparous library, and there were no significant difference in expression between two libraries. This result was ascribed to the same physiological phase (follicular phase) in ovaries of two experimental groups. MiR-21 was verified in regulation of apoptosis in vivo, and related with ovulation rate [46]. However, the expression of miR-21 also have no significant difference between multiple and uniparous groups in this study. Then, we turned our attention to the specific expressed miRNAs. The highest specific expressed miRNA in multiple library was miR-29c, and the one in uniparous library was miR6406. As aligning the clean reads to the miRNA precursor/ mature miRNAs of all animals in the miRBase 20.0 database, and obtained miRNA with no specifid species. Carefully analyzed miR-29c and miR-6406 in the present study, we found that the sequence of miR-29c was consistent with ola-mir-29c (from Oryzias latipes), the sequence of miR-6406 endured some mismatch with mmu-mir6406 (from Mus musculus). miR-29c was in the same family with MiR-29a, which was significantly down-regulated after $12 \mathrm{~h}$ FSH treatment, while its expression increased after $48 \mathrm{~h} \mathrm{FSH}$ treatment [47]. Therefore, it was supposed that miR-29c was also related with FSH secretion which may influence on follicles recruiting in the present study, and it need further experimentation certainly. As for miR6406 , there is no research reported on ovary so far and miRBase recorded the mmu-mir-6406 by reference the article of David [48]. In consideration of specific and higher level expression of miR-6406 in uniparous group, some further experimentation also worth to be done.

GO annotation and KEGG Pathway analyses are able to obtain a better understanding from the cellular components, molecular functions and biological processes of target genes [10]. Start with GO analysis, miRNA targets were significantly enriched to different terms in two libraries, and the significantly enriched terms in uniparous library contained the terms in multiple library. As for KEGG pathway analysis, it is worth to note that Focal adhesion, $\mathrm{ABC}$ transporters, Carbohydrate digestion and absorption, Peroxisome, Starch and sucrose metabolism, and Progesterone-mediated oocyte maturation were involved in the significantly enriched pathway in the ovaries during follicular phase of multiple goats. These significantly enriched pathways may imply that the organism was coping to the criteria of follicular phase in multiple goats. GO annotation and KEGG Pathway analyses can provide a reference to us for the later research.

RT-PCR was carried out to analyze the expression of 5 randomly selected miRNAs in multiple and uniparous hircine ovaries during follicular phase, and the results 
were consistent with the Solexa sequencing data. However, the expression levels of every miRNA need to be validated by RT-PCR in theory. Hence, the identified miRNAs in the present study can only be regard as a hircine ovary-specific miRNA reference dataset. Compared with previous study implemented by Zhang et al. in our team [13], which the ovaries tissues were from different physiological phase of pregnant and non-pregnant phase in the same goats, while the ovaries tissues in this study were from different goat of multiple and uniparous goats in the same follicular phase. Therefore, the results of our previous study were caused by the different physiological phase, while the results of our present study were caused by the different genetic background. Nevertheless, the potential of kidding rate is affected by many components, including ovulation rate, fertilization rate and embryo survival, any or all of which may be under genetic control [49], and these need to be researched step by step.

\section{Conclusions}

In summary, 1008 miRNAs were co-expressed, 309 and 433 miRNAs were specifically expressed in the ovaries of multiple and uniparous goats during follicular phase. The highest specific expressed miRNA in multiple library was miR-29c, and the highest specific expressed miRNA in uniparous library was miR-6406. 35 novel miRNAs were predicted in total. GO annotation and KEGG Pathway analyses were implemented on target genes of all miRNA in two libraries, Progesterone-mediated oocyte maturation and other pathways were pointed out for significantly enriched. The result may help to further understand the role of miRNAs in kidding rate regulation and also help to identify miRNAs which could be potentially used to increase hircine ovulation rate and kidding rate in the future.

\section{Methods}

Animals and sample preparation

The experimental goats of this study, Anhui White goats (a Chinese indigenous breed) were obtained from the College of Animal Science and Technology, Anhui Agricultural University, Hefei, China. The ovaries of Anhui White goats were collected and froze in liquid nitrogen instantly then stored at $-80^{\circ} \mathrm{C}$ for generating small RNA libraries. 6 target goats, 3 were 3 -year old multiple goats whose litter size was more than one (Mul) and the other were 3-year old uniparous goats whose litter size was only one (Uni), accepted the teasing behavior were chosen as our experimental samples for their ovaries. Mul: the three goats had three litters which kidding $\geq 2$. Uni: the three goats had three litters which kidding $=1$. All the experimental procedures with Anhui White goats used in the present study had been given prior approval by the ethics committee of
Anhui Agricultural University, Anhui, China, under permit No. AHAU20101025.

\section{Small RNA library construction and sequencing}

Two groups of total RNA were used for library preparation and sequencing by pooling equal quantity $(10 \mu \mathrm{g})$ of total RNA isolated from six individual multiple and uniparous goats ovaries. Small RNA fragments of 18-30 nt in length were isolated and purified from total RNA using 15\% denaturing polyacrylamide gel electrophoresis (PAGE). Subsequently, a 3' RNA adaptor and 5' RNA adaptor were ligated to the RNA pool using T4 RNA ligase, then the samples were used as templates for cDNA synthesis. The cDNAs were amplified using the appropriate number of PCR cycles to produce sequencing libraries, which were subsequently subjected to the proprietary Solexa sequencing-by-synthesis method using the Illumina Genome Analyzer (SanDiego, CA, USA) at the Beijing Genomics Institute (BGI, Shenzhen, China).

\section{Sequence analysis}

According to the requirement of this experiment and the principle of bioinformatics analysis, some contaminant reads should be removed from the raw reads, such as low quality reads and reads with 5' primer contaminants, reads without 3' primer, reads without the insert tag, reads with poly (A), and reads shorter than $18 \mathrm{nt}$. Then the final clean reads for summarizing the length distribution and counts were got, all valid sequences were remained for further analysis. The clean reads were compared with the ncRNAs (rRNAs, tRNAs, snRNAs, and snoRNA) deposited in the NCBI GenBank database and the Rfam10.1 database using BLAST to annotate the sRNA sequences. The clean reads were also mapped to the goat genome (http://goat.kiz.ac.cn/GGD/download.htm) by SOAP v1.11 to analysis their expression and distribution in the goat genome. The clean reads was aligned to the miRNA precursor/ mature miRNA of all animals in miRBase 20.0 (http://www. mirbase.org/), show the sequence and count of miRNA families (no specific species) which can be found in the samples. According to the characteristic hairpin structure of miRNA precursor can be used to predict novel miRNA. The unannotated sequences were used to predict potential novel miRNA candidates by Mireap (http://sourceforge. net/projects/mireap/) mapped to the goat genome.

\section{Differential expression analysis of two libraries}

Comparing the known miRNA expression between two libraries (Mul and Uni) to find out the differentially expressed miRNAs, Log2-ratio figure and Scatter Plot were plotted. Procedures were shown as below: (1)Normalize the expression of miRNA in two libraries to get the expression of transcript per million (TPM). Normalization formula: Normalized expression $=$ Actual miRNA count $/$ Total count 
of clean reads*1000000; (2) Calculate fold-change and $\mathrm{P}$-value from the normalized expression. Then generate the Log2-ratio figure and Scatter Plot. Fold-change formula:

$$
\text { Fold-change }=\log _{2}(\mathrm{Mul} / \mathrm{Uni})
$$

P-value formula:

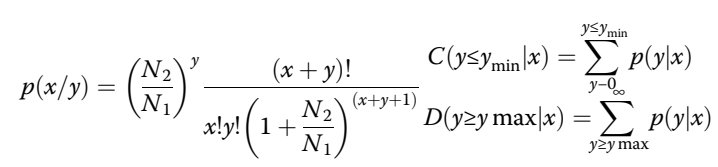

The $\mathrm{x}$ and $\mathrm{y}$ represent normalized expression level, and the N1 and N2 represent total count of clean reads of a given miRNA in small RNA library of ovaries of multiple and uniparous goats, respectively [35].

When the normalized expression of a certain miRNA was zero in one of the two libraries, its expression value was revised to 0.01 . If the normalized expression of a certain miRNA in two libraries was all lower than 1 , further differential expression analysis was conducted without this miRNA for the reason of its low expression.

\section{GO enrichment and KEGG pathway analyses}

GO enrichment analysis of present study was the best on predicted target gene candidates of all detected miRNAs compared to the reference gene background, as well as the genes corresponding to certain biological function. The result could reveal the functions significantly related with predicted target gene candidates of all detected miRNAs. This method firstly mapped all target gene candidates to GO terms in the database (http://www.geneontology. org/), calculated gene numbers for each term, then used hyper geometric test to find significantly enriched GO terms in target gene candidates compared to the reference gene background. The calculating formula is:

$$
P=1-\sum_{i=0}^{m-1} \frac{\left(\begin{array}{c}
M \\
i
\end{array}\right)\left(\begin{array}{c}
N-M \\
n-i
\end{array}\right)}{\left(\begin{array}{c}
N \\
n
\end{array}\right)}
$$

In the formula above, $\mathrm{N}$ is the number of all genes with $\mathrm{GO}$ annotation; $\mathrm{n}$ is the number of target gene candidates in $\mathrm{N} ; \mathrm{M}$ is the number of all genes that were annotated to a certain GO term; $m$ is the number of target gene candidates in M. The Bonferroni Correction for the $\mathrm{p}$-value was used to obtain a corrected p-value. GO terms with corrected $\mathrm{p}$-value $\leq 0.05$ are defined as significantly enriched in target gene candidates. This analysis could recognize the main biological functions for target gene candidates.

The same as Gene Ontology, KEGG pathway analysis is also based on the target gene candidates. In organisms, genes usually interact with each other to play different roles in certain biological function. KEGG pathway analysis could facilitate the understanding of biological functions of genes. KEGG is a major public pathwayrelated database [50]. KEGG pathway analysis identifies significantly enriched metabolic pathways or signal transduction pathways in target gene candidates comparing with the whole reference gene background. The calculating formula is the same as that in $\mathrm{GO}$ analysis. Here $\mathrm{N}$ is the number of all genes with KEGG annotation, $n$ is the number of target gene candidates in N, M is the number of all genes annotated to a certain pathway, and $\mathrm{m}$ is the number of target gene candidates in $\mathrm{M}$. Genes with FDR $\leq 0.05$ are considered as significantly enriched in target gene candidates. The KEGG analysis could reveal the main pathways which the target gene candidates are involved in.

\section{MiRNA validation via RT-PCR}

For validating the Solexa sequencing data, RT-PCR assay was carried out by five randomly selected miRNAs. One microgram of total RNA from each sample were reversetranscript into cDNA using the miScript Reverse Transcription Kit (Qiagen, Dusseldorf, Germany) according to the manufacturer's instructions. The template for RT-PCR was got, after incubation at $37^{\circ} \mathrm{C}$ for $1 \mathrm{~h}$ and deactivation at $95^{\circ} \mathrm{C}$ for $5 \mathrm{~min}$. The reaction system of RT-PCR contained $2.0 \mu \mathrm{l}$ cDNA, $32.5 \mu \mathrm{l}$ SYBRGreen Mix (Thermo, Shanghai, China), $0.5 \mu \mathrm{l}$ of each primer and $14.5 \mu \mathrm{l} \mathrm{H}_{2} \mathrm{O}$. RT-PCR was performed using standard protocols on the Roche LightCycler 480 II Real-Time PCR Detection System (Roche; LC480 II, Basel, Switzerland). The reaction was incubated at $95^{\circ} \mathrm{C}$ for $10 \mathrm{~min}$, followed by 40 cycles of $95^{\circ} \mathrm{C} 15 \mathrm{~s}$ and $60^{\circ} \mathrm{C} 45 \mathrm{~s}$. All reactions were performed in triplicate. The threshold cycle $(\mathrm{CT})$ was collected from each reaction, and the relative expression level of each miRNA to 5S snRNA was evaluated using the equation $2^{\text {-(CTmiRNA-CT5SRNA) }}$. GLM was used to examine the significance of the expression in two samples by SAS 8.0 software. The miRNA specific primers were presented in Additional file 10.

\section{Additional files}

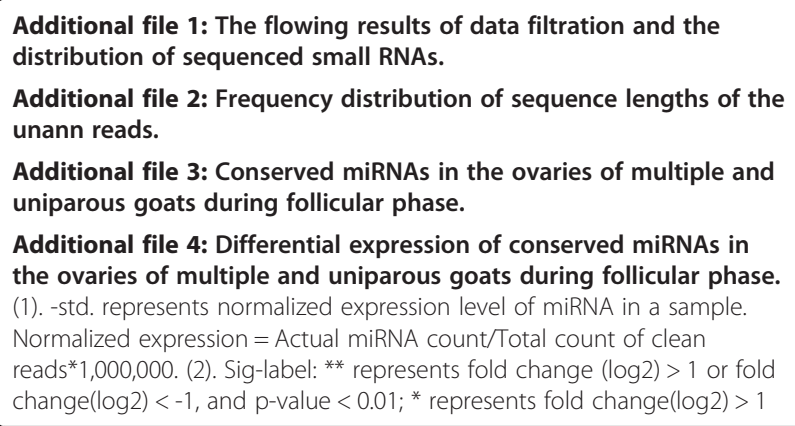

Additional file 3: Conserved miRNAs in the ovaries of multiple and uniparous goats during follicular phase.

Additional file 4: Differential expression of conserved miRNAs in the ovaries of multiple and uniparous goats during follicular phase. (1). -std. represents normalized expression level of miRNA in a sample. Normalized expression $=$ Actual miRNA count/Total count of clean reads ${ }^{*} 1,000,000$. (2). Sig-label: ** represents fold change $(\log 2)>1$ or fold change $(\log 2)<-1$, and $p$-value $<0.01$; ${ }^{*}$ represents fold change $(\log 2)>1$ 
or fold change $(\log 2)<-1$, and $0.01 \leq p<0.05$; None represents others. Fold change $=\log 2$ (Pregnant std./Non-pregnant std.) (3). miRNAs in red font used for the RT-PCR analysis.

Additional file 5: Clustering of miRNAs differentially expressed during follicular phase in ovaries.

Additional file 6: Information of the potential novel miRNAs on goat. Additional file 7: The stem loop structures of precursors of predicted miRNA candidates.

Additional file 8: GO enrichment analysis for the target genes of conserved miRNAs.

Additional file 9: KEGG pathways for the target genes of all detected miRNAs.

Additional file 10: Primer sequences for RT-PCR experiments.

\section{Abbreviations}

miRNA: microRNA; HF: Hair follicle; sRNA: Small RNA; UTR: Untranslated regions; GO: Gene ontology; KEEG: Kyoto encyclopedia of genes and genomes; RT-PCR: Reverse transcription PCR; BGI: Beijing genomics institute; MFE: Minimum free energy; TPM: Transcript per million; GLM: General linear model; SAS: Statistical analysis system.

\section{Competing interests}

The authors declare that they have no competing interests.

\section{Authors' contributions}

These studies were designed by YHL, CHR and ZJZ. YHL and CHR carried out all the experimental analyses and prepared all figures and tables. YHL and XFG analyzed the data and drafted the manuscript. LNX, YFH, JCL, YHZ and XRZ contributed to revisions of the manuscript. ZJZ and XRZ assisted in explaining the results and revised the final version of the manuscript. All authors have read and approved the final manuscript.

\section{Acknowledgments}

This research was supported by the National Natural Science Foundation of China (31301934, 31372310), the China Agriculture Research System funds (No. 11004986), the Natural Science Foundation of Anhui Province (1308085QC54) and the Specific Funds of Public Service Research No. 201303145). We are grateful to Hao Xiang for construction of small RNA libraries.

\section{Author details}

${ }^{1}$ College of Animal Science and Technology, Anhui Agricultural University, No. 130 Changjiang west road, Hefei 230036, P.R. China. ${ }^{2}$ Anhui Provincial Laboratory of Local Animal Genetic Resources Conservation and Biobreeding, No. 130 Changjiang west road, Hefei 230036, P.R. China. ${ }^{3}$ Institute of Plant Protection and Agro-Products Safety, Anhui Academy of Agricultural Sciences, No. 40 South Nongke Road, Hefei 230031, P.R. China.

Received: 8 February 2014 Accepted: 30 April 2014

Published: 6 May 2014

\section{References}

1. Bartel DP: MicroRNAs: genomics, biogenesis, mechanism, and function. Cell 2004, 116(2):281-297.

2. Hu SJ, Ren G, Liu JL, Zhao ZA, Yu YS, Su RW, Ma XH, Ni H, Lei W, Yang ZM: MicroRNA expression and regulation in mouse uterus during embryo implantation. J Biol Chem 2008, 283(34):23473-23484.

3. Hwang HW, Mendell JT: MicroRNAs in cell proliferation, cell death, and tumorigenesis. Br J Cancer 2006, 94(6):776-780.

4. Ivey KN, Srivastava D: MicroRNAs as regulators of differentiation and cell fate decisions. Cell Stem Cell 2010, 7(1):36-41.

5. Ambros V: The functions of animal microRNAs. Nature 2004, 431(7006):350-355.

6. Jansson MD, Lund AH: MicroRNA and cancer. Mol Oncol 2012, 6(6):590-610.

7. Cuellar TL, McManus MT: MicroRNAs and endocrine biology. J Endocrinol 2005, 187(3):327-332.

8. Rottiers $\mathrm{V}$, Näär AM: MicroRNAs in metabolism and metabolic disorders. Nat Rev Mol Cell Biol 2012, 13(4):239-250.
9. Hawkins SM, Buchold GM, Matzuk MM: Minireview: the roles of small RNA pathways in reproductive medicine. Mol Endocrinol 2011, 25(8):1257-1279.

10. Ji Z, Wang G, Xie Z, Wang J, Zhang C, Dong F, Chen C: Identification of novel and differentially expressed microRNAs of dairy goat mammary gland tissues using Solexa sequencing and bioinformatics. PLoS One 2012, 7(11):e49463.

11. Liu Z, Xiao H, Li H, Zhang Y, Lai S, Yu X, Cai T, Du C, Zhang W, Li J: Identification of conserved and vovel microRNAs in cashmere goat skin by deep sequencing. PLoS One 2012, 7(12):e50001.

12. Ling YH, Ding JP, Zhang XD, Wang LJ, Zhang YH, Li YS, Zhang ZJ, Zhang XR: Characterization of microRNAs from goat (Capra hircus) by Solexa deep-sequencing technology. Genet Mol Res 2013, 12(2):1951.

13. Zhang XD, Zhang YH, Ling YH, Liu Y, Cao HG, Yin ZJ, Ding JP, Zhang XR: Characterization and differential expression of microRNAs in the ovaries of pregnant and non-pregnant goats (Capra hircus). BMC Genomics 2013, 14(1):157.

14. Lin X, Luo J, Zhang L, Wang W, Gou D: MiR-103 controls milk fat accumulation in goat (Capra hircus) mammary gland during lactation. PLoS One 2013, 8(11):e79258.

15. Kim HJ, Cui XS, Kim EJ, Kim WJ, Kin NH: New porcine microRNA genes found by homology search. Genome 2006, 49(10):1283-1286.

16. Ro S, Song R, Park C, Zheng H, Sanders KM, Yan W: Cloning and expression profiling of small RNAs expressed in the mouse ovary. RNA 2007, 13(12):2366-2380.

17. Otsuka M, Zheng M, Hayashi M, Lee JD, Yoshino O, Lin S, Han J: Impaired microRNA processing causes corpus luteum insufficiency and infertility in mice. J Clin Invest 2008, 118(5):1944.

18. Menchaca A, Pinczak A, Rubianes E: Follicular recruitment and ovulatory response to FSH treatment initiated on day 0 or day 3 postovulation in goats. Theriogenology 2002, 58(9):1713-1721.

19. Messinis IE, Templeton AA: The importance of follicle-stimulating hormone increase for folliculogenesis. Hum Reprod 1990, 5(2):153-156.

20. Van der Meer M, Hompes PGA, Scheele F, Schoute E, Veersema S, Schoemaker J: Endocrinology: Follicle stimulating hormone (FSH) dynamics of low dose step-up ovulation induction with FSH in patients with polycystic ovary syndrome. Hum Reprod 1994, 9(9):1612-1617.

21. Ganguly A, Meur SK, Ganguly I: Changes in circulatory FSH of Barbari goats following treatment with high molecular weight inhibin isolated from buffalo follicular fluid. Res Vet Sci 2013, 95(2):374-380.

22. Juengel JL, Hudson NL, Whiting L, McNatty KP: Effects of immunization against bone morphogenetic protein 15 and growth differentiation factor 9 on ovulation rate, fertilization, and pregnancy in ewes. Biol Reprod 2004, 70(3):557-561.

23. Orisaka M, Jiang JY, Orisaka S, Kotsuji F, Tsang BK: Growth differentiation factor 9 promotes rat preantral follicle growth by up-regulating follicular androgen biosynthesis. Endocrinology 2009, 150(6):2740-2748.

24. Hanrahan JP, Gregan SM, Mulsant P, Mullen M, Davis GH, Powell R, Galloway SM: Mutations in the genes for oocyte-derived growth factors GDF9 and BMP15 are associated with both increased ovulation rate and sterility in Cambridge and Belclare sheep (Ovis aries). Biol Reprod 2004, 70(4):900-909.

25. Galloway SM, McNatty KP, Cambridge LM, Laitinen MPE, Juengel JL, Jokiranta TS, McLaren RJ, Luiro K, Dodds KG, Montgomery GW, Beattie AE, Davis GH, Ritvos O: Mutations in an oocyte-derived growth factor gene (BMP15) cause increased ovulation rate and infertility in a dosage-sensitive manner. Nat Genet 2000, 25(3):279-283.

26. Cummins $\sqcup$, O'shea T, Bindon BM, Lee WWK, Findlay JK: Ovarian inhibin content and sensitivity to inhibin in Booroola and control strain Merino ewes. J Reprod Fertil 1983, 67(1):1-7.

27. McBride D, Carre W, Sontakke SD, Hogg CO, Law A, Donadeu FX, Clinton M: Identification of miRNAs associated with the follicular-luteal transition in the ruminant ovary. Reproduction 2012, 144(2):221-233.

28. Yao G, Yin M, Lian J, Tian H, Liu L, Li X, Sun F: MicroRNA-224 is involved in transforming growth factor-beta-mediated mouse granulosa cell proliferation and granulosa cell function by targeting Smad4. Mol Endocrinol 2010, 24:540-551.

29. Murchison EP, Stein P, Xuan Z, Pan H, Zhang MQ, Schultz RM, Hannon GJ: Critical roles for Dicer in the female germline. Genes Dev 2007, 21(6):682-693

30. Lin F, Li R, Pan Z, Zhou B, Yu D, Wang X, Ma X, Han J, Shen M, Liu H: miR-26b promotes granulosa cell apoptosis by targeting ATM during follicular atresia in porcine ovary. PLoS One 2012, 7(6):e38640. 
31. Sheedy FJ, Palsson-McDermott E, Hennessy EJ, Martin C, O'Leary JJ, Ruan Q, Jojnson DJ, Chen Y, O'Neill LAJ: Negative regulation of TLR4 via targeting of the proinflammatory tumor suppressor PDCD4 by the microRNA miR-21. Nat Immunol 2010, 11(2):141-147.

32. Xie SS, Li XY, Liu T, Cao JH, Zhong Q, Zhao SH: Discovery of porcine microRNAs in multiple tissues by a Solexa deep sequencing approach. PLoS One 2011, 6(1):e16235.

33. Calabrese JM, Seila AC, Yeo GW, Sharp PA: RNA sequence analysis defines Dicer's role in mouse embryonic stem cells. Proc Natl Acad Sci U S A 2007, 104(46):18097-18102.

34. Hao DC, Yang L, Xiao PG, Liu M: Identification of Taxus microRNAs and their targets with high-throughput sequencing and degradome analysis. Physiol Plant 2012, 146(4):388-403.

35. Huang J, Ju Z, Li Q, Hou Q, Wang C, Li J, Li R, Wang L, Sun T, Hang S, Gao Y, Hou M, Zhong J: Solexa sequencing of novel and differentially expressed microRNAs in testicular and ovarian tissues in Holstein cattle. Int J Biol Sci 2011, 7(7):1016.

36. Yuan C, Wang X, Geng R, He X, Qu L, Chen Y: Discovery of cashmere goat (Capra hircus) microRNAs in skin and hair follicles by Solexa sequencing. BMC Genomics 2013, 14(1):511.

37. Zhang Y, Zhou X, Ge X, Jiang J, Li M, Jia S, Yang X, Kan Y, Miao G, Zhao G, Li F, Huang Y: Insect-specific microRNA involved in the development of the silkworm Bombyx mori. PLoS One 2009, 4(3):e4677.

38. Schneider MR: MicroRNAs as novel players in skin development, homeostasis and disease. Br J Dermatol 2012, 166(1):22-28.

39. Mardaryev AN, Ahmed MI, Vlahov NV, Fessing MY, Gill JH, Sharov AA, Botchkareva NV: Micro-RNA-31 controls hair cycle-associated changes in gene expression programs of the skin and hair follicle. FASEB J 2010, 24(10):3869-3881.

40. Easow G, Teleman AA, Cohen SM: Isolation of microRNA targets by miRNP immunopurification. RNA 2007, 13(8):1198-1204.

41. Mascarenhas R, Simöes Nunes A, Robalo Silva J: Cyclic reproductive activity and efficiency of reproduction in Serrana goats. Anim Reprod Sci 1995, 38(3):223-229.

42. Khanum SA, Hussain M, Kausar R: Assessment of reproductive parameters in female Dwarf goat (Capra hircus) on the basis of progesterone profiles. Anim Reprod Sci 2007, 102(3):267-275.

43. Zhang CY, Chen SL, Li X, Xu DQ, Zhang Y, Yang LG: Genetic and phenotypic parameter estimates for reproduction traits in the Boer dam. Livest Sci 2009, 125(1):60-65.

44. Bradford GE, Quirk JF, Famula TR: Fertility, embryo survival and littex size in lines of Targhee sheep selected for weaning weight or titter size. J Anim Sci 1986, 62:395.

45. Scaramuzzi RJ, Adams NR, Baird DT, Campbell BK, Downing JA, Findlay JK, Henderson KM, Martin GB, MCNatty KP, McNeilly AS, Tsonis CG: A model for follicle selection and the determination of ovulation rate in the ewe. Reprod Fertil Dev 1993, 5(5):459-478.

46. Carletti MZ, Fiedler SD, Christenson LK: MicroRNA 21 blocks apoptosis in mouse periovulatory granulosa cells. Biol Reprod 2010, 83(2):286-295.

47. Yao N, Yang BQ, Liu Y, Tan XY, Lu CL, Yuan XH, Ma X: Follicle-stimulating hormone regulation of microRNA expression on progesterone production in cultured rat granulosa cells. Endocrine 2010, 38(2):158-166.

48. Humphreys DT, Hynes CJ, Patel HR, Wei GH, Cannon L, Fatkin D, Suter CM, Clancy $J$, Preiss T: Complexity of murine cardiomyocyte miRNA biogenesis, sequence variant expression and function. PLOS One 2012, 7(2):e30933.

49. Schoenian SG, Burfening PJ: Ovulation rate, lambing rate, litter size and embryo survival of Rambouillet sheep selected for high and low reproductive rate. J Anim Sci 1990, 68(8):2263-2270.

50. Kanehisa M, Araki M, Goto S, Hattori M, Hirakawa M, Itoh M, Katayama T, Kawashima S, Okuda S, Tokimatsu T, Yamanishi Y: KEGG for linking genomes to life and the environment. Nucleic Acids Res 2008, 36(suppl 1):D480-D484.

doi:10.1186/1471-2164-15-339

Cite this article as: Ling et al:: Identification and characterization of microRNAs in the ovaries of multiple and uniparous goats (Capra hircus) during follicular phase. BMC Genomics 2014 15:339.

\section{Submit your next manuscript to BioMed Central and take full advantage of:}

- Convenient online submission

- Thorough peer review

- No space constraints or color figure charges

- Immediate publication on acceptance

- Inclusion in PubMed, CAS, Scopus and Google Scholar

- Research which is freely available for redistribution

Submit your manuscript at www.biomedcentral.com/submit
C Biomed Central 